\title{
O tabagismo e o transtorno do pânico: gravidade e comorbidades
}

\author{
Smoking and panic disorder: severity and comorbidities
}

\author{
Rafael Christophe da Rocha Freire', Marco André Mezassalma², Alexandre Martins Valença ${ }^{3}$, \\ Valfrido Leão de-Melo-Neto ${ }^{4}$, Fabiana Leão Lopes $^{5}$, Isabella Nascimento ${ }^{6}$, Antônio Egidio \\ Nardi $^{7}$
}

${ }^{1}$ Médico psiquiatra. Mestrando, Instituto de Psiquiatria, Universidade Federal do Rio de Janeiro (UFRJ), Rio de Janeiro, RJ. ${ }^{2}$ Mestre em Psiquiatria. Doutorando, Instituto de Psiquiatria, UFRJ. ${ }^{3}$ Doutor em Psiquiatria. Professor adjunto, Departamento de Psiquiatria, Universidade Federal Fluminense (UFF), Niterói, RJ. ${ }^{4}$ Médico psiquiatra. Mestrando, Instituto de Psiquiatria, UFRJ. ${ }^{5}$ Doutora em Psiquiatria. Pesquisadora, Instituto de Psiquiatria, UFRJ. ${ }^{6}$ Doutora em Psiquiatria. Pesquisadora, Instituto de Psiquiatria, UFRJ. ${ }^{7}$ Doutor em Psiquiatria. Professor adjunto, Instituto de Psiquiatria, UFRJ.

Este estudo foi realizado no Laboratório do Pânico e Respiração, Instituto de Psiquiatria (IPUB), Universidade Federal do Rio de Janeiro (UFRJ), Rio de Janeiro, RJ.

Pesquisa financiada pelo Conselho Nacional de Desenvolvimento Científico e Tecnológico (CNPq).

\section{Resumo}

Introdução: Estudos indicam que há uma associação entre tabagismo e transtorno do pânico, e alguns autores sugerem que o tabagismo aumenta o risco de ataques de pânico e transtorno do pânico. Este estudo analisa a hipótese de que pacientes fumantes com esse transtorno apresentam um quadro clínico mais grave.

Método: Sessenta e quatro pacientes em tratamento no Laboratório do Pânico e Respiração (Instituto de Psiquiatria da Universidade Federal do Rio de Janeiro), com transtorno do pânico, segundo critérios do Manual de Diagnóstico e Estatística das Perturbações Mentais (DSM, $4^{\mathrm{a}}$ edição), foram divididos em grupos de tabagistas e não-tabagistas. Os grupos foram avaliados quanto a características sociodemográficas, comorbidades e gravidade do quadro clínico.

Resultados: Não houve diferença significativa em relação à gravidade do transtorno do pânico; no entanto, tabagistas tiveram prevalência de depressão significativamente maior $(\mathrm{p}=0,014)$ do que não-tabagistas.

Conclusão: Este estudo não evidenciou que o transtorno do pânico em tabagistas é mais grave, porém indicou que esses pacientes têm mais comorbidade com depressão.

Descritores: Transtorno de pânico, agorafobia, tabagismo, transtorno depressivo.

\begin{abstract}
Introduction: Several studies indicate that panic disorder and tobacco smoking are associated, and some authors hypothesize that smoking increases the risk of panic attacks and panic disorder. The objective of this study is to investigate whether smokers have a more severe form of panic disorder than non-smokers.

Method: Sixty-four patients already in treatment at the Laboratory of Panic and Respiration (Instituto de Psiquiatria da Universidade Federal do Rio de Janeiro) with panic disorder as established by the Diagnostic and Statistical Manual of Mental Disorders, fourth edition, were divided into groups of smokers and non-smokers. Both groups were compared regarding sociodemographic data, comorbidities and clinical status severity.

Results: There was no statistically significant difference between the two groups regarding severity; however, prevalence of depression was significantly higher in the smoker group than in non-smokers $(p=0.014)$.

Conclusion: This study did not indicate that smokers have a more severe form of panic disorder, but smoking and comorbid depression were associated.
\end{abstract}

Keywords: Panic disorder, agoraphobia, smoking, depressive disorder.

\section{Correspondência:}

Rafael Christophe da Rocha Freire, Laboratório do Pânico e Respiração, UFRJ, Rua Visconde de Pirajá, 407/702, CEP 22410-003, Rio de Janeiro, RJ. Fax: (21) 2523-6839. E-mail: rafaelcrfreire@terra.com.br. 


\section{Introdução}

Estudos recentes indicam que há uma prevalência elevada de tabagismo em pacientes com transtornos psiquiátricos como esquizofrenia, depressão, transtorno bipolar do humor, transtorno do pânico (TP), transtorno obsessivo-compulsivo, transtorno de estresse pós-traumático e transtorno do déficit de atenção com hiperatividade ${ }^{1}$. Essa associação foi mais estudada em relação aos transtornos de ansiedade e, entre eles, os ataques de pânico (AP) e o TP parecem estar mais intimamente ligados ao hábito de fumar ${ }^{2,3}$. Estudos epidemiológicos indicam que a prevalência de tabagismo entre pacientes com TP é maior que a da população em geral ${ }^{4,5}$ e que, na maior parte dos casos, o tabagismo precede o surgimento do TP, sugerindo que o uso de tabaco poderia ser um fator de risco para AP e $\mathrm{TP}^{4-9}$. Em contrapartida, outros estudos indicam que o TP não aumenta o risco de tabagismo ${ }^{5-7}$ e que, possivelmente, os sintomas do TP motivariam as pessoas a parar de fumar ${ }^{4}$.

Em um estudo realizado com 2.032 adolescentes australianos, foi evidenciado um maior risco de tabagismo em indivíduos cujos pares fumavam ou que apresentavam sintomas de ansiedade ou depressão ${ }^{10}$. Duas possíveis explicações para essa associação seriam: o tabagismo e os transtornos de ansiedade compartilham uma mesma etiologia; indivíduos ansiosos teriam um risco maior para o tabagismo ${ }^{10}$. Alguns autores levantaram a hipótese de que há um grupo de tabagistas que usa a nicotina para obter sedação, e outro que usa essa substância pelo efeito estimulante ${ }^{11}$, mas estudos mais recentes indicam que a nicotina tem efeitos estimulantes e sedativos nos mesmos indivíduos ${ }^{12}$. Também foi verificado que, quando o uso de cigarros ocorre simultaneamente a um estímulo distraidor, há uma redução da ansiedade, porém, caso esse estímulo não esteja presente, o nível de ansiedade não se altera significativamente ${ }^{13}$. A nicotina leva a um aumento da atividade adrenérgica, com aumento da freqüência cardíaca, vasoconstricção, elevação da pressão arterial e aumento da motilidade intestinal, e em doses altas poderia levar a $\mathrm{AP}^{14}$. Um estudo epidemiológico conduzido por Breslau \& Klein ${ }^{6}$, com 5.418 indivíduos, indicou que o uso diário de tabaco aumenta significativamente o risco para AP e TP. Diversos estudos evidenciaram, ainda, que o TP está relacionado a anormalidades do sistema respiratório ${ }^{15-}$ ${ }^{18}$ e que o hábito de fumar poderia ser um dos mecanismos causadores dessas anormalidades ${ }^{6-8}$. West \& Hajek ${ }^{19}$ estudaram 101 fumantes com critérios para dependência de nicotina pelo Manual de Diagnóstico e Estatística das Perturbações Mentais - $4^{\text {a }}$ edição (DSM-IV) que interromperam o uso de cigarros. Encontraram aumento da ansiedade logo antes do cessamento do tabagismo, e em um período de 4 semanas houve redução gradual da ansiedade a níveis mais baixos do que nas 2 semanas que antecederam a cessação do tabagismo ${ }^{19}$. No entanto, há, na literatura, relatos de casos de pacientes com TP que param de fumar e, durante a síndrome de abstinência, apresentam significativo aumento dos $\mathrm{AP}^{20}$.

Zvolensky ${ }^{21}$ estudou o efeito do tabagismo em pacientes com TP. Em pacientes fumantes, foi evidenciada uma maior intensidade de sintomas de ansiedade, maior comprometimento funcional e mais complicações clínicas do que em pacientes nãofumantes ${ }^{21}$. O objetivo deste estudo é confirmar se tabagistas apresentam um quadro clínico do TP mais grave. Esperamos encontrar mais comorbidade com depressão e agorafobia, tempo de doença mais longo e um maior escore de gravidade em pacientes fumantes.

\section{Método}

Este estudo foi conduzido no Instituto de Psiquiatria (IPUB) da Universidade Federal do Rio de Janeiro (UFRJ). O IPUB, que é um hospital psiquiátrico universitário conveniado ao Sistema Único de Saúde, atende predominantemente pacientes da zona sul do Rio de Janeiro, porém grande parte dos pacientes vem de outros locais da cidade, e esses pacientes possuem variados níveis socioeconômicos e culturais. Este estudo foi um corte transversal dos pacientes com TP que estavam em tratamento no Laboratório do Pânico e Respiração do IPUB.

\section{Amostra}

Foi utilizada, neste estudo, uma amostra clínica de conveniência composta por 64 pacientes que, no momento da avaliação, estavam na fase aguda do TP, iniciando ou fazendo o tratamento psiquiátrico há no máximo 6 meses. Fumantes e não-fumantes receberam o mesmo tipo de tratamento. Os diagnósticos foram feitos com o Structured Clinical Interview Diagnostic for Axis I Disorders (SCID-I) 22 para o DSM-IV ${ }^{23}$. Os critérios para inclusão foram idade entre 18 e 65 anos e diagnóstico de TP pelos critérios do DSM-IV. Para manter a homogeneidade da amostra, foram incluídos apenas pacientes que tiveram pelo menos três AP nas 2 semanas que antecederam a entrevista. Entre os indivíduos com depressão maior, distimia ou transtorno de ansiedade generalizada, apenas os que tiveram o diagnóstico principal de TP 
foram incluídos no estudo. Os critérios de exclusão foram retardo mental, transtorno psicótico, transtorno bipolar do humor e patologia clínica grave (incluindo asma grave e doença pulmonar obstrutiva crônica grave). Este estudo foi aprovado pelo comitê de ética do IPUB da UFRJ.

\section{Dados sociodemográficos e clínicos}

Os pacientes foram entrevistados por um psiquiatra e forneceram informações sobre sexo, idade, escolaridade, renda familiar em salários mínimos e se estavam empregados ou não. Também foram questionados em relação ao tempo de duração e idade de início da doença, ao hábito de fumar e a casos de familiares de primeiro grau com TP. Pacientes que pararam de fumar há menos de 1 ano foram considerados fumantes. O psiquiatra avaliou, ainda, a gravidade do quadro clínico no momento da entrevista, classificando-o de acordo com a Escala de Impressão Clínica Global (CGI) ${ }^{24}$ para cada paciente.

\section{Análise estatística}

A amostra foi dividida em dois grupos tabagistas e não-tabagistas -, que foram comparados usando-se os testes $t$ de Student e qui-quadrado. Nos testes em que a amostra não apresentou uma distribuição normal, o teste $t$ de Student foi substituído pelo Mann-Whitney Rank Sum Test. A análise foi bidirecional, e a significância estatística foi estabelecida em 0,05 . O software estatístico utilizado foi o SPSS 13.0.

\section{Resultados}

\section{A amostra}

Na amostra estudada, a média de idade foi de $39,8$ anos (DP $=11,7)$; 46,9\% $(\mathrm{n}=30)$ eram homens, $\mathrm{e}$ $53,1 \%(n=34)$ eram mulheres. A escolaridade foi codificada da seguinte forma: 0 - até fundamental incompleto; 1 - fundamental completo; 2 - médio incompleto; 3 - médio completo; 4 - superior completo. A média de escolaridade da amostra foi de 3,2 (desvio padrão - DP = 1,4), o que seria equivalente a nível médio completo. A idade média de início dos sintomas do TP foi de 31,8 anos (DP =9,8). A média do tempo entre o início dos sintomas e a avaliação foi de 96,9 meses ( $D P=101,2)$. A média do CGI foi de 3,9 (DP = 1,3) (Tabela 1). Dos entrevistados, 34,4\% (n $=22)$ eram tabagistas, sendo que $35,3 \%(n=12)$ das mulheres e $33,3 \%(n=10)$ dos homens fumavam. As mulheres apresentaram uma maior prevalência de comorbidade com depressão $(61,8 \%)$ que os homens (40\%), porém essa diferença não foi estatisticamente significativa (qui-quadrado $=3,023,1 \mathrm{gl}, \mathrm{p}=0,082$ ).

Tabela 1 - Características da amostra

\begin{tabular}{lcc}
\hline & Média (DP) & \% (n) \\
\hline Sexo (feminino) & $53,1(34)$ \\
Idade (anos) & $39,8(11,7)$ & \\
Escolaridade & $3,2(1,4)$ & \\
Empregado & & $73,4(47)$ \\
Renda familiar & $10,2(8,3)$ & \\
Idade de início (anos) & $31,8(9,8)$ & \\
Duração do TP (meses) & $96,9(101,2)$ & \\
Agorafobia & & $76,6(49)$ \\
Depressão & & $51,6(33)$ \\
CGI & $3,9(1,3)$ & \\
Tabagistas & & $34,4(22)$ \\
\hline
\end{tabular}

CGI = Escala de Impressão Clínica Global; DP = desvio padrão; TP = transtorno do pânico.

\section{Comparação entre grupos}

Não houve diferenças sociodemográficas significativas entre o grupo de tabagistas e o de nãotabagistas. As diferenças de idade de início e duração do TP, história familiar, CGI e prevalência de agorafobia também não foram significativas na comparação dos dois grupos. O grupo de tabagistas apresentou uma prevalência de depressão significativamente maior (qui-quadrado $=6,013,1 \mathrm{gl}$, $\mathrm{p}=0,014)$ (Tabela 2, Figura 1).

\section{Discussão e conclusão}

A prevalência de tabagismo na amostra estudada, em ambos os sexos, foi semelhante à da população brasileira, na qual $38 \%$ dos homens e $24 \%$ das mulheres fumam $^{25}$. Entretanto, em pacientes com TP, foi encontrada prevalência de tabagismo um pouco maior para mulheres e um pouco menor para os homens do que na população em geral, o que poderia ser atribuído a características específicas dos pacientes com TP. A associação entre tabagismo e depressão e a elevada prevalência de depressão em mulheres com TP poderiam explicar a alta prevalência de tabagismo entre mulheres na amostra estudada. 
Tabela 2 - Comparação entre não-tabagistas e tabagistas

\begin{tabular}{lccc}
\hline & Não-tabagistas $(\mathbf{n}=\mathbf{4 2})$ & Tabagistas $(\mathbf{n}=\mathbf{2 2})$ & $\mathbf{p}$ \\
\hline Sexo (feminino) \% (n) & $52,4(22)$ & $54,5(12)$ & $\mathrm{NS}$ \\
Idade média (DP) & $39,6(11,1)$ & $40,1(13,1)$ & $\mathrm{NS}$ \\
Escolaridade média (DP) & $3,1(1,4)$ & $3,3(1,5)$ & $\mathrm{NS}$ \\
Empregado \% (n) & $71,4(30)$ & $77,3(17)$ & $\mathrm{NS}$ \\
Renda familiar média (DP) & $9,0(8,0)$ & $12,3(8,7)$ & $\mathrm{NS}$ \\
Idade de início média (DP) & $32,6(10,1)$ & $30,1(9,2)$ & $\mathrm{NS}$ \\
Duração do TP média (DP) & $84,8(98,3)$ & $119,9(104,9)$ & $\mathrm{NS}$ \\
História familiar de TP \% (n) & $38,1(16)$ & $40,9(9)$ & $\mathrm{NS}$ \\
Agorafobia \% (n) & $71,4(30)$ & $86,4(19)$ & $\mathrm{NS}$ \\
Depressão \% (n) & $40,5(17)$ & $72,7(16)$ & 0,014 \\
CGI média (DP) & $3,8(1,4)$ & $4,0(1,0)$ & $\mathrm{NS}$ \\
\hline
\end{tabular}

CGI = Escala de Impressão Clínica Global; DP = desvio padrão; NS = não-significativo $(p>0,100)$; TP = transtorno do pânico.

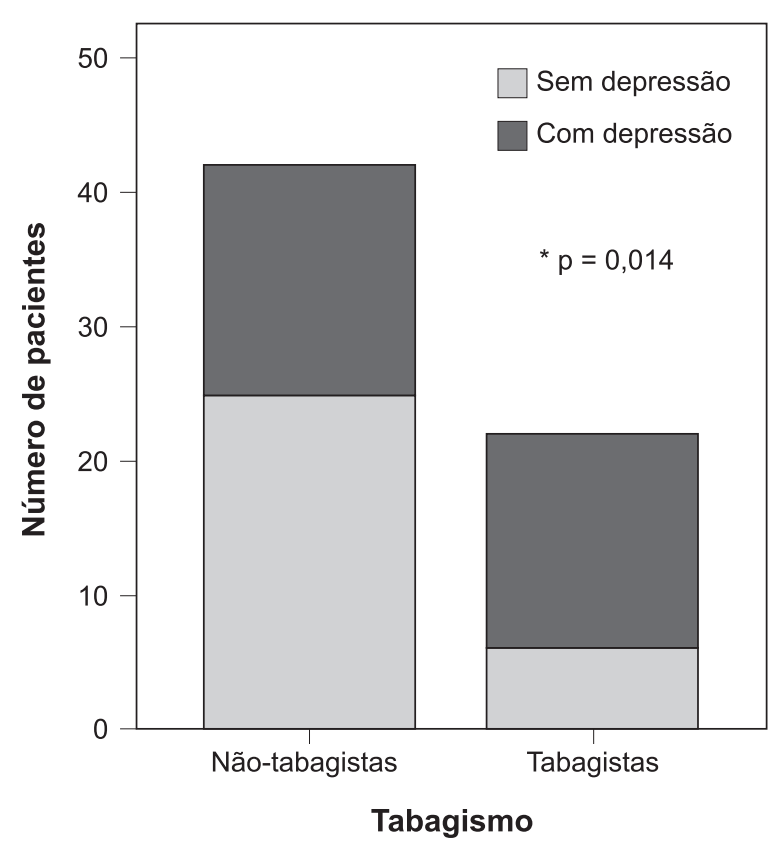

Figura 1 - Prevalência de depressão em tabagistas e não-tabagistas

Zvolensky et al. ${ }^{21}$ encontrou que fumantes com TP apresentavam mais ansiedade, maior ansiedade antecipatória em relação a AP e maior gravidade do TP, porém não foi encontrado aumento significativo de freqüência ou intensidade dos AP nesses pacientes. No estudo atual, não foi encontrada diferença estatisticamente significativa da gravidade do TP na comparação entre tabagistas e não-tabagistas.
A prevalência de agorafobia foi similar entre tabagistas e não-tabagistas, e foi encontrada uma associação significativa entre tabagismo e depressão. No estudo de Zvolensky et al. ${ }^{21}$ também não houve diferenças em relação à agorafobia, mas os escores de depressão e a prevalência de transtorno depressivo foram similares entre os dois grupos. Há relatos de alta incidência de tabagismo em pacientes com $\mathrm{TP}^{1-3,26}$, em pacientes com depressão $0^{1,26-31}$ e em pacientes com comorbidades $^{26}$, porém a associação de TP com depressão e tabagismo é um novo achado. Acredita-se que a nicotina provoca redução dos afetos negativos, redução do estresse e aumento dos afetos positivos, e isso se dá pela ativação do nucleus accumbens e outros núcleos dopaminérgicos centrais envolvidos no sistema de recompensa ${ }^{31}$. Esse efeito positivo da nicotina explica porque pessoas com depressão têm maior risco para o tabagismo $^{32}$. Por outro lado, a abstinência à nicotina leva a um aumento dos afetos negativos, humor deprimido, nervosismo, inquietação, irritabilidade, ansiedade, impaciência, raiva, agressividade, sonolência e sensação de fadiga ${ }^{28}$, por isso tabagistas com depressão têm mais dificuldade para parar de fumar e apresentam mais recaídas no uso de tabaco ${ }^{31}$.

Este estudo possui uma série de limitações, como o pequeno tamanho da amostra, pacientes em diferentes estágios do tratamento e avaliação da gravidade feita apenas com o CGI. A maior parte das pesquisas que estuda a associação entre TP e tabagismo usa amostras populacionais e aleatórias, e este estudo utilizou uma amostra clínica de conveniência e de menor tamanho. Possivelmente, com uma amostra maior, poderiam evidenciar-se outras associações, como a relação entre tabagismo e gravidade. Todos os pacientes estavam na 
fase aguda do TP e receberam o mesmo tratamento, porém alguns já apresentavam alguma melhora dos sintomas, enquanto outros ainda estavam iniciando o tratamento, o que pode ter influenciado os escores do CGI. Não foram utilizadas escalas como Beck Anxiety Index ${ }^{33}$, Panic Disorder Severity Scale ${ }^{34}$ e Sheehan Patient-Rated Anxiety Scale ${ }^{35}$, usadas em outros estudos.

Pacientes com TP tabagistas e não-tabagistas apresentaram perfis sociodemográficos e características clínicas muito semelhantes. Os resultados mostraram que o diagnóstico de TP comórbido com depressão maior estava associado fortemente ao hábito de fumar.

\section{Referências}

1. Kalman D, Morissette SB, George TP. Co-morbidity of smoking in patients with psychiatric and substance use disorders. Am J Addict. 2005;14:106-23.

2. McCabe RE, Chudzik SM, Antony MM, Young L, Swinson RP, Zvolensky MJ. Smoking behaviors across anxiety disorders. J Anxiety Disord. 2004;18:7-18.

3. Morissette SB, Brown TA, Kamholz BW, Gulliver SB. Differences between smokers and nonsmokers with anxiety disorders. J Anxiety Disord. 2006;20:597-613.

4. Amering M, Bankier B, Berger P, Griengl H, Windhaber J, Katschnig H. Panic Disorder and Cigarette Smoking Behavior. Compr Psychiatry. 1999;40:35-8.

5. Isensee B, Wittchen HU, Stein MB, Hofler M, Lieb R. Smoking increases the risk of panic: findings from a prospective community study. Arch Gen Psychiatry. 2003;60:692-700.

6. Breslau N, Klein DF. Smoking and panic attacks. Arch Gen Psychiatry. 1999;56:1141-7.

7. Johnson JG, Cohen P, Pine DS, Klein DF, Kasen S, Brook JS Association between cigarette smoking and anxiety disorders during adolescence and early adulthood. JAMA. 2000;284:234851.

8. Goodwin RD, Lewinsohn PM, Seeley JR. Cigarette smoking and panic attacks among young adults in the community: the role of parental smoking and anxiety disorders. Biol Psychiatry. 2005;58:686-93.

9. Bernstein A, Zvolensky MJ, Schmidt NB, Sachs-Ericcson N. Developmental course(s) of lifetime cigarette use and panic attack comorbidity: an equifinal phenomenon? Behav Modif. 2007;31:117-35.

10. Patton GC, Carlin JB, Coffey C, Wolfe R, Hibbert M, Bowes G. Depression, anxiety, and smoking initiation: a prospective study over 3 years. Am J Public Health. 1998;88:1518-22.

11. Russell MA. Subjective and behavioral effects of nicotine in humans: some sources of individual variation. In: Nordberg A, Fuxe
K, Holmstedt B, Sundwall A, editors. Progress in brain research. New York: Elsevier; 1989. p. 289-302.

12. West R. Dr. West replies. Am J Psychiatry. 1999;156:336.

13. Kassel JD, Shiffman S. Attentional mediation of cigarette smoking's effect on anxiety. Health Psychol. 1997;16:359-68.

14. Dilsaver SC. Nicotine and panic attacks. Am J Psychiatry. 1987;144:1245-6

15. Klein DF. Testing the suffocation false alarm theory of panic disorder. Anxiety. 1994;1:1-7.

16. Biber B, Alkin T. Panic disorder subtypes: differential responses to CO2 challenge. Am J Psychiatry. 1999;156:739-44.

17. Nascimento I, Nardi AE, Valença AM, Lopes FL, Mezzasalma MA, Nascentes R, et al. Psychiatric disorders in asthmatic outpatients. Psychiatry Res. 2002;110:73-80.

18. Caldirola D, Bellodi L, Caumo A, Migliarese G, Perna G. Approximate entropy of respiratory patterns in panic disorder. Am J Psychiatry. 2004;161:79-87.

19. West R, Hajek P. What happens to anxiety levels on giving up smoking? Am J Psychiatry. 1997;154:1589-92.

20. Valença AM, Nascimento I, Nardi AE. Smoking and panic disorder. Psychiatr Serv. 2001;52:1105-6.

21. Zvolensky MJ, Schmidt NB, McCreary BT. The impact of smoking on panic disorder: An initial investigation of a pathoplastic relationship. J Anxiety Disord. 2003;17:447-60.

22. First MB, Spitzer RL, Gibbon M, Williams JB. Structured Clinical Interview Diagnostic (SCID) for DSM-IV axis I disorders: clinician version (SCID-CV). Washington: American Psychiatric; 1997.

23. American Psychiatric Association. Diagnostic and Statistical Manual for Mental Disorders. 4th edition (DSM-IV). Washington: American Psychiatric; 1994.

24. Guy W, editors. ECDEU Assessment Manual for Psychopharmacology. Rockville: NIMH; 1976.

25. Instituto Brasileiro de Geografia e Estatística. Pesquisa nacional sobre saúde e nutrição: dados preliminares. Rio de Janeiro: IBGE; 1989.

26. Lopes FL, Nascimento I, Zin WA, Valença AM, Mezzasalma MA, Figueira I, et al. Smoking and psychiatric disorders: a comorbidity survey. Braz J Med Biol Res. 2002;35:961-7.

27. Mathew RJ, Weinman ML, Mirabi M. Physical symptoms of depression. Br J Psychiatry. 1981;139:293-6.

28. Hughes JR, Hatsukami DK, Mitchell JE, Dahlgren LA. Prevalence of smoking among psychiatric outpatients. Am J Psychiatry. 1986;143:993-7.

29. Anda RF, Williamson DF, Escobedo LG, Mast EE, Giovino GA, Remington PL. Depression and the dynamics of smoking. JAMA. 1990;264:1583-4.

30. Glassman AH. Cigarette smoking: implications for psychiatric illness. Am J Psychiatry. 1993;150:546-53.

31. Hall SM, Munoz RF, Reus VI. Nicotine, negative affect and depression. J Consult Clin Psychol. 1993;61:761-7.

32. Kandel DB, Davies M. Adult sequelae of adolescent depressive symptoms. Arch Gen Psychiatry. 1986;43:255-62.

33. Beck AT, Epstein N, Brown G, Steer RA. An inventory for measuring clinical anxiety: psychometric properties. J Consult Clin Psychol. 1988;56:893-7.

34. Shear MK, Brown TA, Barlow DH, Money R, Sholomskas DE, Woods SW, et al. Multicenter collaborative Panic Disorder Severity Scale. Am J Psychiatry. 1997;154:1571-5.

35. Sheehan DV. The anxiety disease. New York: Scribers; 1983. 